\title{
Le partenariat avec le secteur économique, tendances et perspectives en Allemagne
}

Katharina D. Giesel et Julia Mann

\section{(2) OpenEdition}

12 Journals

Édition électronique

URL : http://journals.openedition.org/ere/6606

DOI : $10.4000 /$ ere.6606

ISSN : 2561-2271

Éditeur

Centr'ERE

Référence électronique

Katharina D. Giesel et Julia Mann, « Le partenariat avec le secteur économique, tendances et perspectives en Allemagne », Éducation relative à l'environnement [En ligne], Volume 3 | 2002, mis en ligne le 20 mars 2002, consulté le 16 avril 2021. URL : http://journals.openedition.org/ere/6606 ; DOI : https://doi.org/10.4000/ere.6606

Ce document a été généré automatiquement le 16 avril 2021. 


\title{
Le partenariat avec le secteur économique, tendances et perspectives en Allemagne
}

\author{
Katharina D. Giesel et Julia Mann
}

1 Durant les quinze dernières années, l'éducation à l'environnement s'est énormément développée dans le secteur non formel allemand. La promotion et le soutien financier dont elle a bénéficié de la part d'institutions telle que la Deutsche Bundesstiftung Umwelt (Fondation Fédérale Allemande pour l'Environnement - DBU) ${ }^{1}$ ont contribué à cette expansion.

2 Afin de faire évaluer l'état des lieux ainsi que les perspectives d'avenir de l'éducation non formelle à l'environnement en Allemagne, la DBU a initié une vaste enquête quantitative et qualitative qui a été confiée au service de recherche en éducation à l'environnement à la Freie Universität Berlin (Université Libre de Berlin). Touchant toutes les institutions de l'éducation à l'environnement (EE) dans le secteur non formel, l'enquête a révélé la situation ainsi que les champs d'innovation possibles dans ce secteur.

3 Les résultats de l'évaluation étant trop divers (Giesel, de Haan et Rode, 2001; Giesel et coll., 2001), nous nous contenterons ici d'identifier le secteur économique et les entreprises comme des partenaires prometteurs de l'éducation à l'environnement non formelle, d'appuyer notre argumentation sur les résultats empiriques du sondage ainsi que sur une réflexion à propos des tendances actuelles au niveau social et théorique.

\section{L'éducation non formelle et ses partenaires}

\section{L'enquête}

4 L'enquête a révélé que le nombre d'établissements s'investissant dans l'éducation à l'environnement en dehors des structures d'enseignement formel en Allemagne s'élève à plus de 4600. Environ 2850 de ces établissements ont contribué à l'enquête (tableau 1) 
en fournissant des informations détaillées sur la forme et le fond de leurs offres (méthodologies appliquées, thèmes traités, etc.). Ils nous ont renseigné sur leur public, l'équipement et la situation de leurs établissements, les structures internes et financières, le personnel, leur intégration régionale ainsi que sur leurs partenaires et les formes de coopération réalisées. Finalement ils nous ont donné leurs avis sur l'avenir probable de l'éducation à l'environnement.

Tableau 1 : Les différentes parties de l'enquête

\begin{tabular}{|l|c|c|}
\hline \multicolumn{1}{|c|}{ Partie de l'enquête } & Méthode appliquée & Échantillon \\
\hline Questionnaire des institutions & standardisé & $\begin{array}{c}2856 \\
\text { (61\% du nombre } \\
\text { total estimé) }\end{array}$ \\
\hline $\begin{array}{l}\text { Questionnaire des acteurs / } \\
\text { animateurs pédagogiques }\end{array}$ & standardisé \\
\hline $\begin{array}{l}\text { Questionnaire des participants } \\
\begin{array}{l}\text { Questionnaire du grand public } \\
\text { et de 50 professeurs }\end{array}\end{array}$ & standardisé & 353 \\
\hline $\begin{array}{l}\text { Entretiens avec des acteurs et } \\
\text { partenaires }\end{array}$ & interview sémi-ouvert \\
\hline $\begin{array}{l}\text { Dialogue avec les acteurs et } \\
\text { les institution d'administration }\end{array}$ & $\begin{array}{l}\text { 2 ateliers d'avenir, } \\
6 \text { cercles de qualités }\end{array}$ & $350+50$ \\
\hline
\end{tabular}

Parallèlement à cette enquête quantitative auprès des établissements, nous avons interviewé 600 acteurs pédagogiques de l'éducation à l'environnement, 400 personnes appartenant à la population ordinaire, et presque autant de participants à des manifestations d'éducation à l'environnement. De plus, nous avons mené des entretiens avec 90 experts de l'éducation à l'environnement.

Pour l'interprétation des données qualitatives, nous avons eu recours à un concept d'analyse développé par le service de recherche en éducation à l'environnement: l'analyse des imaginations-guide (Leitbildanalyse). Elle sert à l'identification des représentations qui sous-tendent les discours. En structurant la perception, elles focalisent la pensée et sont déterminantes pour la prise de décision et le comportement des individus. Les imaginations-guides résument les idées et buts qu'une communauté a en partage et peuvent donc servir à définir les communautés et leurs organisations ${ }^{2}$. Les imaginations-guides circulant dans le secteur non formel de l'éducation à l'environnement ont été d'une importance primordiale pour l'enquête évaluative puisqu'elles renseignent sur les schémas d'orientation et les visions d'avenir des acteurs.

\section{Qu'est-ce que l'éducation à l'environnement non formelle ?}

7 L'éducation à l'environnement non formelle a été définie comme comprenant toutes les activités éducatives liées à l'environnement des institutions et des organisations se situant en dehors des structures formelles d'enseignement (écoles, collèges ou lycées d'enseignement général ou professionnel, universités). Les établissements de l'éducation à l'environnement non formelle se composent donc d'associations de protection de nature et de l'environnement, de centres ou maisons de la nature et de l'environnement, d'établissements d'éducation populaire (Volkshochschulen), de musées, d'associations diverses, d'académies de partis politiques et d'Églises, d'administrations, 
de ligues de consommateurs, de Chambres de commerce, d'entreprises, de grands centres de recherche et autres organismes ou institutions concentrant la totalité de leurs activités sur l'éducation à l'environnement ou encore ne s'engagent que partiellement dans ce domaine (tableau 2).

Tableau 2 : Typologie des institutions

\begin{tabular}{|r|c|}
\hline & Institutions \\
\hline Association de protection de la nature ou de l'environnement & $21,1 \%$ \\
\hline Maison de la nature ou de l'environnement & $13,6 \%$ \\
\hline Association & $10,7 \%$ \\
\hline Établissement de l'éducation populaire & $10,6 \%$ \\
\hline Administration & $8,9 \%$ \\
\hline Entreprise & $6,3 \%$ \\
\hline Science et recherche & $4,9 \%$ \\
\hline Musée & $4,1 \%$ \\
\hline Chambre de commerce et d'industrie & $3,4 \%$ \\
\hline Ligue de l'enseignement & $3,1 \%$ \\
\hline Fondation & $2,6 \%$ \\
\hline Autres* & $10,8 \%$ \\
\hline $\mathrm{N}=$ & 2839 \\
\hline
\end{tabular}

${ }^{*} \mathrm{Y}$ inclus les institutions de moins de 2,5\% quote-part (académie, ligue de consommateurs, formation professionnelle, Église, partie politique).

Dans une analyse différenciée, Giesel (1999) a démontré qu'il n'y a pas de rapport étroit entre les réflexions théoriques plutôt abstraites sur l'éducation à l'environnement et les considérations plutôt pragmatiques ou les orientations de base des acteurs. Il n'y a même pas parmi les acteurs un accord minimal sur ce qu'est l'éducation à l'environnement.

De ce fait, il est primordial de ne pas restreindre le champ d'étude à partir d'une définition de l'éducation à l'environnement. La culture du quotidien est un « modèle du premier ordre " à partir duquel la science développe un " modèle de second ordre " (Lamnek, 1995); on en déduit que l'éducation à l'environnement est ce que les acteurs pensent qu'elle est ${ }^{3}$.

Les institutions de l'éducation à l'environnement sont d'une variété énorme (tableau 2 ; dans cette multitude de types d'établissements se distinguent par leur nombre les associations de protection de la nature ou de l'environnement, les centres de la nature et de l'environnement, les associations en général, les établissements de l'éducation populaire ainsi que les institutions administratives.

11 Cette variété institutionnelle du secteur non formel est démultipliée par les offres thématiques, les types de manifestations et les méthodes d'éducation à l'environnement. L'éducation à l'environnement comprend des interventions pédagogiques directes (telles que des séminaires ou des cours d'information) et des éléments divers d'information, d'illustration et de conseil (excursions, conférences, expositions, consultations écologiques). S'y ajoutent des champs d'activité comme la mise en réseau des acteurs du processus d'Agenda 21 local, la collaboration à l'élaboration de plans de développement régional, la réalisation de campagnes et les activités de protection de la nature. 

coopérer avec d'autres institutions (84,1\%). Quand on parle de coopération ou de partenariat, on n'entend pas toujours par là un partenariat dans le sens d'une relation d'échange sur pied d'égalité ou d'activités réalisées en commun. Souvent, on entend par coopération et partenariat un quelconque service unilatéral offert à une autre institution (tableau 3).

Tableau 3 : Les domaines de la coopération

\begin{tabular}{|r|c|}
\hline & Institutions \\
\hline Réalisation commune de manifestations & $84,9 \%$ \\
\hline Échange d'animateurs & $45,1 \%$ \\
\hline Consultation réciproque & $42,7 \%$ \\
\hline Publications communes & $36,6 \%$ \\
\hline Consultation pour éviter des doublements thématiques \\
dans les programmes & $27,1 \%$ \\
\hline Réalisation de projets internationaux & $26,7 \%$ \\
\hline Partage du bureau & $12,9 \%$ \\
\hline $\mathrm{N}=$ & 2403 \\
\hline
\end{tabular}

15 La plupart de ces établissements indiquent qu'ils réalisent des manifestations en commun avec d'autres institutions. Cette forme de partenariat ainsi que l'échange d'enseignants et la consultation réciproque sont certainement la majorité des offres faites à d'autres institutions. En effet, ce sont souvent des écoles ou encore des maternelles qui figurent parmi les soi-disant partenaires (mais que l'on devrait plutôt compter parmi le public de l'éducation à l'environnement non formelle). Par contre, lorsque les partenaires cités sont des communes, on peut s'attendre à plus de réciprocité dans l'échange et dans la réalisation de certaines activités (tableau 4). 
Tableau 4 : Avec qui coopérez-vous ?

\begin{tabular}{r|c}
\hline & Institutions \\
\hline Institutions non européennes & $4,6 \%$ \\
\hline Académies pour la formation professionnelle & $13,4 \%$ \\
\hline Institutions européennes & $16,6 \%$ \\
\hline Partis et institutions politiques & $20,6 \%$ \\
\hline Chambres de commerce et d'industrie & $22,9 \%$ \\
\hline Églises & $23,9 \%$ \\
\hline Maternelles & $24,8 \%$ \\
\hline Entreprises & $32,3 \%$ \\
\hline Conseillers écologiques des communes & $40,0 \%$ \\
\hline Autres établissements de l'EE & $48,8 \%$ \\
\hline Écoles & $51,9 \%$ \\
\hline Administrations communales & $52,4 \%$ \\
\hline Associations & $56,6 \%$ \\
\hline Autres & $26,7 \%$ \\
\hline $\mathrm{N}=$ & 2374 \\
\hline & \\
\hline
\end{tabular}

\section{Un nouveau partenaire : le secteur économique} formelle en Allemagne pour focaliser notre attention sur le secteur économique comme nouveau partenaire prometteur de l'éducation à l'environnement. l'éducation à l'environnement quand on leur demande d'imaginer l'avenir. Le partenariat économique est une nouvelle tendance en Allemagne puisqu'il a été cité par bon nombre d'acteurs de l'éducation à l'environnement dont les institutions ne sont pas encore actives dans ce domaine.

Pour pouvoir expliquer pourquoi et comment le secteur économique représente un nouveau partenaire, il faut se rendre compte de trois tendances au niveau sociopolitique et théorique :

- la notion du développement durable qui entraine une réorientation conceptuelle de l'éducation à l'environnement ;

- l'importance croissante de la formation permanente ;

- l'importance de l'offre en éducation à l'environnement eu égard aux restrictions budgétaires de l'État allemand.

\section{Le développement durable}

19 C'est avec le Sommet de la Terre à Rio en 1992 que la notion de développement durable a fait son entrée dans le discours environnemental en Allemagne. La notion du développement durable implique l'élargissement des perspectives sectorielles purement écologiques, sociales ou économiques pour considérer désormais les aspects globaux.

Le débat autour de l'Agenda 21 et du développement durable a provoqué un processus de réorientation et d'innovation dans l'éducation à l'environnement allemande. Depuis 
1995, l'offre thématique a été enrichie de composantes techniques, économiques et sociales. Le cadre méthodologique a été adapté aux nouvelles exigences, et l'éducation à l'environnement s'est transformée du point de vue conceptuel en éducation pour un développement durable (de Haan et Harenberg, 1999).

La typologie recommandée par les auteurs distingue les centres de la nature et les centres d'Agenda 21 et souligne notamment le fait que l'éducation à l'environnement doit faire face à de nouvelles exigences tout en reconnaissant que l'adaptation à cellesci ne concernera qu'une partie des établissements (de Haan et coll., 1997; de Haan et Harenberg, 1999).

\section{La formation permanente}

Depuis que l'éducation et la formation ne peuvent plus être réduites à une phase précise de la vie, la formation permanente devient de plus en plus importante (Arnold et coll., 1994). Elle dépasse ses anciens contours institutionnels, didactiques et normatifs (Arnold et Schüßler, 1998).

23 La diversification institutionnelle devient évidente dans l'offre de l'éducation à l'environnement du secteur non formel : plus d'un tiers des établissements s'engagent principalement dans la formation continue, lui attribuant ainsi une fonction éminente dans l'éducation à l'environnement en Allemagne (Giesel et Rode, 2000).

\section{La concurrence et les restrictions budgétaires}

Depuis les années 1980, années fondatrices de l'éducation à l'environnement en Allemagne, le marché de l'éducation à l'environnement est devenu de plus en plus dense. De nouvelles institutions se sont établies, d'autres - déjà existantes - ont pris de l'extension ou ont intégré l'éducation à l'environnement dans leurs activités.

Les établissements de l'éducation à l'environnement dépendent à un haut degré de capitaux extérieurs, notamment de ressources publiques. Presque deux établissements sur trois reçoivent des fonds publics. À côté de ceux-ci, les dons représentent une source essentielle de financement extérieur tandis que les revenus propres proviennent en général des frais de participation.

Sous la pression de la concurrence et face aux restrictions budgétaires de l'État, les institutions de l'éducation non formelle à l'environnement se voient obligées de trouver de nouveaux débouchés, de cibler de nouveaux groupes et de trouver de nouvelles sources de financement autres que le Trésor Public.

\section{La coopération avec le secteur économique et ses difficultés}

\section{Les différentes formes de coopération}

La coopération avec le secteur économique peut se réaliser sous différentes formes :

- Les entreprises sont des clients potentiels de l'éducation non formelle à l'environnement. Elles représentent donc un groupe-cible pour des offres éducatives. En complétant et 
soutenant l'éducation à l'environnement dans la formation professionnelle et dans l'entreprise, le secteur non formel peut assurer sa propre existence.

- Le secteur économique et administratif vit une nécessité accrue de tenir compte des aspects écologiques : les réglementations environnementales demandent en outre l'introduction de l'éducation à l'environnement dans la formation professionnelle et continue. L'introduction enfin de la certification environnementale pour les entreprises (norme ISO 14000, de l'audit environnemental) a contribué à intensifier l'intérêt du secteur économique pour les questions de durabilité et de viabilité. Outre la formation continue à l'intérieur où à l'extérieur de l'entreprise, l'éducation à l'environnement pourrait intervenir en tant que conseiller environnemental dans les processus de certification. De fait, ce sont là des propositions qui figurent également parmi les souhaits exprimés par les acteurs de l'éducation à l'environnement lors de nos entretiens avec les experts. Les acteurs veulent s'adresser aux PME. Outre l'information sur la protection environnementale en entreprise, les institutions souhaitent intervenir dans la réalisation et la certification des systèmes de management environnemental (suivant le système communautaire de management environnemental et d'audit [EMAS] ou la norme ISO 14000).

- Les entreprises font partie des acteurs régionaux qui sont à intégrer dans les processus d'établissement de plans de développement régional tels que les processus d'Agenda 21 local. Les résultats de l'enquête révèlent que ce champ de coopération est attrayant pour une grande partie des établissements : approximativement trois établissements sur quatre situés dans une commune avec une initiative d'Agenda 21 local disent coopérer avec celle-ci. Il existe donc des convergences qui invitent à la coopération entre le secteur éducatif et celui de l'économie. Les institutions éducatives ne sont pas limitées à leur travail d'éducation à l'environnement, mais peuvent de plus assister à l'élaboration de plans de développement régional, d'autant qu'elles peuvent s'investir dans l'élaboration des Agendas 21 locaux.

- Finalement, les entreprises figurent parmi les partenaires prometteurs de l'éducation à l'environnement quand il s'agit de trouver de nouveaux moyens financiers. Suivant l'enquête, deux établissements sur trois disent que l'organisation à laquelle ils sont rattachés est en même temps leur plus important bailleur de fonds. Être rattaché à une organisation - et celle-ci pourrait aussi bien être une entreprise - représente donc une garantie financière non négligeable. Les dons monétaires, le mécénat d'entreprise constituent d'autres formes pour faire participer le secteur économique au financement de l'éducation à l'environnement. Les fondations figuraient à la tête des sources financières les plus populaires lorsque nous avons demandé aux experts d'imaginer les possibilités d'assurer les revenus de leurs établissements à long terme; le partenariat avec les entreprises sera facilité par le biais d'une fondation.

\section{Les obstacles au partenariat avec le secteur économique}

28 Les acteurs de l'éducation à l'environnement non formelle en Allemagne doivent franchir quelques obstacles pour réussir un partenariat avec le secteur économique. Nos analyses des imaginations-guides révèlent que les acteurs fondent souvent leurs relations de coopération sur des relations personnelles et de confiance. On se connaît, on s'apprécie, on se ressemble, on a des expériences en commun. Ce sont donc plus des relations personnelles que contractuelles qui dominent le champ des coopérations en éducation à l'environnement. 
Apparemment, il n'est pas d'usage, parmi les acteurs de l'éducation à l'environnement, de passer des contrats. Dans les imaginations-guides (Leitbilder) identifiés, il est rare de trouver des représentations de coopération s'appuyant sur des considérations économiques. En général, on s'imagine des coopérations fondées sur la sympathie réciproque. Pour nous, il s'est avéré pertinent, pour l'analyse des attitudes et des activités des acteurs de l'éducation à l'environnement, de faire la différence entre l'orientation sociale et l'orientation rationnelle. Même au-delà des représentations de partenariat, la sympathie et l'affection ont souvent le pas sur le calcul rationnel, les considérations économiques ou objectives.

Afin de communiquer adéquatement avec le secteur économique, les acteurs devraient s'accommoder de la logique économique et accepter le partenariat contractuel.

\section{Conclusion : le secteur économique, un partenaire pour tous?}

Nous avons présenté ci-dessus de multiples variantes qui s'offrent au niveau de la coopération entre le secteur économique et l'éducation non formel à l'environnement. Il est toutefois fortement déconseillé d'en déduire qu'un tel partenariat pourrait être réalisé par tous les établissements de l'éducation à l'environnement.

Pour les établissements travaillant en tant que centre de la nature, ayant un profil d'offre qui s'adresse aux enfants et adolescents ou encore au secteur des loisirs et qui de plus basent et veulent baser leurs partenariats sur la sympathie réciproques, les entreprises resteront des partenaires plutôt insignifiants.

Les entreprises sont avant tout un groupe-cible et un partenaire prometteur des centres d'Agenda 21 futurs ainsi que des établissements concentrant déjà aujourd'hui leurs efforts dans le domaine de la formation permanente.

Le succès de la coopération dépendra principalement de la capacité des acteurs intéressés à intégrer dans leurs imaginations-guides l'idée du partenariat sur base de contrat.

\section{BIBLIOGRAPHIE}

Arnold, R. et Schüßler, I. (1998). Wandel der Lernkulturen. Ideen und Bausteine für ein lebendiges Lernen. Darmstadt : Wissenschaftliche Buchgesellschaft.

Arnold, R., Weiterbildung et Beruf. (1994). In Tippelt et Ru-dolf (dir.), Handbuch Erwachsenenbildung/Weiterbildung. Opladen : Leske et Budrich. p. 226-236.

Giesel, K.D. (1999). Pädagogische Leitbilder für die außerschulische Umweltbildung. Eine Analyse von Konzepten und Denkmustern. Teil I und II. Berlin : Forschungsgruppe Umweltbildung FU-Berlin, Paper 99-157. 
Giesel, K.D., de Haan, G. et Rode, H. (2001). Umweltbildung in Deutschland. Stand und Trends im außerschulischen Bereich. Heidelberg/New York.

Giesel, K.D., de Haan, G., Rode, H., Schröter et Witte. (2001). Außerschulische Umweltbildung. In Zahlen (dir.), Die Evaluationsstudie der Deutschen Bundesstiftung Um-welt. Berlin.

Giesel, K.D. et Rode, H. (2000). Berufliche Umweltbildung im außerschulischen Bereich - eine Bestandsaufnahme. In M. Härtel, R. Stockmann et H. Gaus (dir.), Berufliche Umweltbildung und Umweltberatung. Grundlagen, Konzepte und Wirkungsmessung. Berlin. p. 176-191.

de Haan, G. (2001). Die Leitbildanalyse. Ein Instrument zur Erfassung zukunftsbezogener Orientierungsmuster. In de Haan, G., Lantermann, Linneweber, Reusswig (dir.), Typenbildung in der sozialwissenschaftlichen Umweltforschung. Opladen. p. 69-106.

de Haan, G. et Harenberg, D. (1999). Bildung für eine nachhaltige Entwicklung. Bonn : Bund-LänderKommission für Bildungsplanung und Forschungsförderung. Materialien zur Bildungsplanung und zur Forschungsförderung Heft 72.

de Haan et coll. (1997). Umweltbildung als Innovation. Bilanzierung und Empfehlungen zu Modellversuchen und Forschungsvorhaben. Berlin/Heidelberg : Springer.

Lamnek. (1995). Qualitative Sozialforschung. Bd. 1 : Methodologie, 3. korr. Aufl., Weinheim.

\section{NOTES}

1. La DBU finance et subventionne des projets novateurs visant la sauvegarde de l'environnement.

2. Pour plus d'informations sur la méthode d'analyse, voir de Haan (2001).

3. Notre sondage demandait aux interviewés de s'inclure ou de s'exclure eux-mêmes. Ceux qui trouvaient dans le questionnaire des liens avec leurs activités étaient comptés comme faisant partie de l'éducation à l'environnement.

\section{AUTEURS}

\section{KATHARINA D. GIESEL}

Katharina D. Giesel est assistante scientifique au service de recherche sur l'éducation à l'environnement de la Freie Universität Berlin où elle s'investit dans la publication des résultats de l'évaluation de l'éducation à l'environnement non formelle en Allemagne. Ses activités de recherche sont prioritairement orientées vers la communication des risques, le constructivisme radical et l'analyse des imaginations-guides (Leitbildanalyse) qui est aussi au centre de sa thèse de Doctorat en cours de réalisation.

\section{JULIA MANN}

Julia Mann collabore avec le service de recherche sur l'éducation à l'environnement de la Freie Universität Berlin. Elle est spécialiste des pratiques de l'éducation au développement durable en 
Europe. Sa maîtrise de fin d'études traite de la théorie en éducation à l'environnement et de la comparaison de l'évolution des discours théoriques en Allemagne et en France depuis 1992. 